\title{
Los grupos de interés y sus efectos sobre las políticas públicas: el caso del FUT
}

\author{
Mónica Ruiz*
}

\begin{abstract}
Resumen
Los grupos de interés conforman un tipo de acción colectiva que ha sido estudiada ampliamente durante los últimos años, debido a la influencia que ejercen sobre las políticas públicas que se quieran establecer en un determinado momento. En Chile su influencia quedó demostrada durante la negociación de la reforma tributaria por parte del gobierno de la Nueva Mayoría, donde el oficialismo no logró sacar la reforma tal como estaba propuesta en el programa de gobierno; en particular, el tema del Fondo de Utilidades Tributables, conocido como FUT, sufrió grandes modificaciones. Especial relevancia tomaron los sectores empresariales, los que realizaron una intensa campaña para modificar los principales aspectos de la normativa.
\end{abstract}

Palabras clave: Grupos de interés - acción colectiva - FUT - reforma tributaria.

\begin{abstract}
Interest groups form a kind of collective action that has been widely studied in recent years due to their influence on public policy. In Chile its influence was demonstrated during the negotiation of tax reform by the government of New Majority. In this case, the government failed to take the reform as it was proposed in the government program. Indeed the issue of Taxable Income Fund -known as FUTunderwent major modifications. Special relevance took the entrepreneurial sectors, who conducted an intensive campaign in order to change the main aspects of the legislation.
\end{abstract}

Keywords: Interest groups - collective action - FUT- tax reform.

* Ingeniero Comercial, mención Economía, Pontificia Universidad Católica de Chile. Master of Arts in Development Economics, Sussex University, Inglaterra. Tesista Magíster en Ciencia Política, Universidad de Chile. 


\section{INTRODUCCIÓN}

En el programa de gobierno de la presidenta Bachelet se establece la necesidad de realizar una reforma tributaria cuyo principal objetivo es recaudar US\$ 8.200 millones, lo que representa aproximadamente el 3\% del Producto Interno Bruto (PIB). Estos recursos, de naturaleza permanente, se destinarán a financiar gastos de la misma naturaleza que se comprometerán, principalmente, en la reforma educacional.

"Para recaudar esta cantidad es necesario no solo modificar ciertas cargas tributarias, sino avanzar en medidas que disminuyan la evasión y la elusión" (Programa de Gobierno de Michelle Bachelet 2014-2018: 24). Es en este contexto donde se considera la eliminación del Fondo de Utilidades Tributables (FUT), ya que, por la naturaleza de esta normativa, su eventual eliminación se percibe como una herramienta que permitirá aumentar los niveles de recaudación al disminuir la elusión y evasión fiscal. Una vez ganadas las elecciones, el 1 de abril de 2014, el gobierno ingresó al Congreso un proyecto de reforma tributaria, el que fue rápidamente cuestionado desde sectores de la oposición, pero también por parte de algunos integrantes de la Nueva Mayoría (NM).

Sin embargo, uno de los actores más relevantes en la discusión antes señalada fueron los grupos de interés, en particular los grupos relacionados al sector empresarial. Truman, en 1962, los definió como asociaciones o grupos de personas que comparten un mismo interés y desarrollan actividades destinadas a representar y defender ese interés particular. Como característica distintiva se puede señalar que poseen capacidad organizativa.

En este documento se muestra un ejemplo de la capacidad organizativa y de la influencia que pueden ejercer los grupos de interés: el caso de la reforma tributaria y en particular los aspectos relacionados al FUT. En este contexto, los grupos empresariales, reunidos en diversas asociaciones como la Confederación de la Producción y del Comercio (CPC), la Sociedad de Fomento Fabril (SOFOFA) o la Asociación de Emprendedores, entre otras, influyeron en el resultado final de la reforma, el que distó mucho de ser el planteado originalmente por el gobierno, que contemplaba la total eliminación del FUT. ¿Por qué sucedió esto? ¿Cómo afectó la intervención de los grupos de interés en este resultado?

El planteamiento central que aquí se expone es que los grupos de interés, representados principalmente por las asociaciones que agrupan a los grandes, medianos y pequeños empresarios, hicieron diversas gestiones y campañas que permitieron que la comunicación y las acciones realizadas por el gobierno en relación con la reforma tributaria, y en particular al FUT, no lograran penetrar en la población, dificultándose, a su vez, la coordinación con los partidos de la NM. De esta manera, se generó un espacio para que se realizaran cambios profundos en la iniciativa al introducir nuevas temáticas que terminaron por modificar los argumentos iniciales que sustentaban a la reforma. Para comenzar se describirá brevemente cómo la participación social se ha ido ampliando a lo largo del tiempo y cuáles son las expresiones y acciones específicas de cada una de sus principales categorías. Finalmente, se verá cuál es la capacidad que poseen los grupos de interés en particular para influir en las decisiones políticas, ejemplificando con el caso específico del FUT. 


\section{LA AMPLIACIÓN DE LA PARTICIPACIÓN SOCIAL: DEL VOTO INDIVIDUAL A LA ACCIÓN COLECTIVA}

A medida que las sociedades se desarrollan tanto socialmente como respecto de sus instituciones democráticas, se generan diversos espacios para la expresión ciudadana, de manera que a los partidos políticos, que es la manera más clásica y estudiada de participación, se le suman los grupos de interés y los movimientos sociales. Esta evolución no solo implica la ampliación de la participación democrática ciudadana en términos de voto para elegir a sus representantes, sino que abre una serie de opciones de participación que de otra manera no lograrían emerger. En este sentido, la democracia no sería solo un constructo realizado por las instituciones gubernamentales, sino que es necesaria la participación ciudadana más allá de los procesos electorales (Merino, 1995).

En la antigüedad la democracia directa solo era posible gracias al pequeño tamaño de las ciudades-Estado y al limitado número de personas que ostentaban el título de ciudadano. Con el tiempo, el concepto y la práctica evolucionaron hasta la introducción de la democracia representativa y los procesos electorales ${ }^{1}$ (Merino, 1995). Pero la participación ciudadana en una democracia representativa no debería limitarse solamente al voto. En efecto, los ciudadanos participan más allá de los periodos eleccionarios cuando estos sienten que no están siendo representados adecuadamente o sus demandas no quedan cubiertas de manera satisfactoria por las acciones Ilevadas a cabo por parte de los representantes electos. Es en la mezcla de las libertades que otorga la democracia y en la capacidad de los ciudadanos de exigir más de sus representantes, y del sistema en general, que emergen maneras "adicionales" de participación como los grupos de interés y los movimientos sociales.

Sin embargo, algunos autores como Dalton, Wattenberg y Lijphart miran con preocupación el alejamiento de las personas respecto de los partidos políticos y la baja participación en las votaciones -participación tradicional- debido a la desigualdad que pueda producirse respecto de la influencia política del grupo que vota o pertenece a un partido político en relación con quienes no realizan estas acciones (Dalton y Wattenberg, 2000; Lijphart, 1996). Lamentablemente las cifras son congruentes con esta apreciación: en la última elección presidencial solo votó el 51,7\% del total de la población en edad de votar, cifra consistente con la tendencia que venía observándose. En cuanto a la militancia política, según cifras de 2012 , solo el $5,1 \%$ de los chilenos pertenecen a un partido político y la mayoría de quienes sí lo hacen tienen más de 40 años. No obstante, la preferencia por la democracia, que es otra posible lectura de los bajos niveles de participación, no cae y se mantiene alta en Chile: la encuesta 2013 de Latinobarómetro muestra que al consultarle a la ciudadanía si "la democracia es preferible a cualquier otra forma de gobierno", el 63\% de la población responde de manera afirmativa. Es decir, la baja participación política tradicional no es sinónimo de una baja preferencia por la democracia y no refleja, necesariamente, una baja participación definida en términos más amplios.

1 En su artículo, Merino destaca la idea de que la democracia, tal como la entendemos en la actualidad, sigue siendo un privilegio de algunos pocos países. 
En relación con lo anteriormente señalado, un estudio del Instituto Libertad y Desarrollo muestra que durante el 2012 solo en la Región Metropolitana hubo 57 manifestaciones, cifra que aumenta a 99 durante 2013. Durante el 2012 participó en ellas, aproximadamente, el $21 \%$ de la población de la Región Metropolitana y el 14,5\% de la población de regiones. Respecto de la participación específica en grupos de interés, como ejemplo se puede ver que solamente la SOFOFA reúne a 2.500 empresas, 30 asociaciones sectoriales y 8 gremios empresariales regionales, lo que refleja un grado interesante de involucramiento ciudadano en formas "menos tradicionales" de participación.

De aquí la importancia de estudiar estas otras instancias de participación y la manera en que los ciudadanos se expresan e influyen políticamente por medio de ellas de manera continua en el tiempo, condicionando al sistema político más allá de los periodos puramente eleccionarios. Particularmente interesante resulta el estudio de los grupos de interés debido a la influencia y visibilidad que han logrado.

\section{III. ¿QUÉ ES UN GRUPO DE INTERÉS?: ORGANIZACIÓN E INFLUENCIA}

Para estudiar los grupos de interés es necesario diferenciarlos de otras formas de participación ciudadana, como son los partidos políticos y los movimientos sociales, con los cuales comparten algunos atributos y se diferencian en otros. $Y$ aunque en la práctica estas diversas instancias de participación se presentan de manera poco clara, siendo difícil distinguir una de otra, en la teoría es posible tipificar cada una claramente. Como se señaló anteriormente, en 1962 Truman los definió como asociaciones o grupos de personas que comparten un mismo interés y desarrollan actividades destinadas a representar y defender ese interés particular.

De manera general, tanto los grupos de interés como los movimientos sociales y los partidos políticos se ocupan del problema de la acción colectiva. A grueso modo, la teoría de la acción colectiva establece que personas con los mismos intereses y metas se agruparán para lograr la concreción de ellos (Olson, 1992). Sin embargo esta lógica se ve afectada por las acciones individuales motivadas por los intereses particulares, las que no siempre coinciden con las acciones grupales. No obstante, de alguna manera intereses colectivos, individuales, objetivos compartidos y medios se aúnan para la realización de acciones colectivas. En el caso de los grupos de interés muchas veces el solo hecho de imponer incentivos $y / o$ sanciones facilita el actuar de manera unitaria como grupo.

Kitschelt (1999) establece una clasificación basada en la estrategia utilizada por cada una de las formas de participación social para enfrentar el problema de la acción colectiva. Para esto distingue el grado de "inversión" en infraestructura organizacional y la amplitud de las demandas y objetivos colectivos de cada una de ellas. De esta manera, los grupos de interés poseen un alto nivel de acción colectiva, por ello invierten en redes comunicacionales, cadenas de mando o cualquier otro aspecto que les permita organizarse de manera eficiente y establecer jerarquías al interior del grupo. Esta inversión se realizará toda vez que la "causa" sea percibida como de largo plazo, pues solo así se obtendrán los retornos deseados. En el caso de los partidos políticos programáticos, la inversión en infraestructura organizacional 
es incluso más alta y extrema. Por último, en el caso de los movimientos sociales esto no es así, ya que sus demandas generalmente aparecen por una causa puntual y la acción colectiva Ilevada a cabo desaparece al acabarse el problema. En cuanto a los niveles de elección social, los grupos de interés no cuentan con ideologías que abarquen una gran cantidad de temas como los partidos políticos, pero sus objetivos son más amplios que los de los movimientos sociales.

En este contexto general, los grupos de interés se diferencian de otras agrupaciones sociales que nacen de manera espontánea por tener un fin y una organización estable y por la existencia de un vínculo permanente entre los asociados que permite que sus miembros se coordinen dentro de la organización para la consecución del fin perseguido. En este sentido se asemejan a los partidos políticos: ambos cuentan con capacidad organizativa formal. Sin embargo, se diferencian de ellos en la medida en que no participan de la contienda electoral. En efecto, los grupos de interés representan intereses particulares y específicos, de manera similar a los movimientos sociales, y se diferencian de los partidos políticos en que no presentan candidatos para puestos de representación popular en las elecciones políticas, cuya misión, una vez electos, es representar el interés general.

En cuanto a las acciones desplegadas, los grupos de interés pueden actuar fuera o dentro del ámbito institucional. Mientras en el primer caso las acciones son realizadas por fuera de los canales formales vinculándose con el sistema político de manera indirecta ${ }^{2}$, como por ejemplo, realizando acciones como protestas o marchas. Sin embargo, típicamente operan dentro de los canales formales existentes, pero manteniéndose fuera del campo electoral. Estas últimas prácticas pueden incluir lobby, informes en sesiones parlamentarias, campañas de presión a políticos concretos, conferencias de prensa públicas, etc. (Kitschelt, 1999: 9). Lachmann (2012) señala que, concretamente, los grupos de interés ofrecen a los políticos ayudas financieras (donaciones), información, conocimiento, asesoría política, dictámenes, cargos en los consejos de administración de empresas o cargos lucrativos al terminar sus trayectorias, entre otras cosas. Pueden "infiltrar" a los partidos y apoyar o perjudicar a los políticos por los medios de comunicación. También pueden aprovechar el recurso de amenazar con restar su apoyo para cierto partido o diputado en las próximas elecciones, cambiar el emplazamiento de sus empresas, etc. (Lachmann, 2012: 6).

No obstante, la mayoría de las acciones desplegadas dentro del ámbito institucional caen en la definición de lobby. Se entiende por lobby a los esfuerzos por cambiar las políticas públicas actuales: "es un esfuerzo por cambiar aspectos sobre lo que ya existe más que realizar un cambio total de la política pública existente" (Baumgartner et al., 2009: 19). Más precisamente, Farnel define el lobby como "una actividad consistente en proceder a intervenir para influenciar directa o indirectamente los procesos de elaboración, aplicación o interpretación de medidas legislativas, normas, reglamentos y, generalizando, de toda

2 En general estas acciones son propias de los movimientos sociales más que de los grupos de interés, aunque, como se explicó anteriormente, ambas formas de acción colectiva comparten varias características, siendo, en ocasiones, difícil distinguir entre unas y otras. 
intervención o decisión de los poderes públicos" (Arrau, 2002: 5). Aunque el lobby es una actividad política intrínsecamente legítima y potencialmente beneficiosa para la sociedad, en el sentido de que en sociedades democráticas las personas tienen el derecho de expresar a sus autoridades sus orientaciones respecto de ciertos temas y de aportar en la discusión legislativa de los mismos, debe ser regulada debido a los posibles efectos negativos en términos de transparencia e integridad que pueda tener sobre las personas que finalmente toman las decisiones, y en general la influencia que ejerce sobre todo el proceso de toma de las mismas.

Tilly acuña el término repertorio para referirse a "un modelo en el que la experiencia acumulada de los actores se entrecruza con las estrategias de las autoridades, dando como resultado un conjunto de medios de acción limitados, más práctico, más atractivo y más frecuente que muchos otros medios que podrían, en principio, servir los mismos intereses" (Martínez e Iranzo, 2010: 199). Este concepto se traduce en un esquema que explica los procesos de transformación de los medios de acción de la lucha política a lo largo del tiempo y si bien este concepto analiza la acción colectiva en general, sin duda es útil para estudiar a los grupos de interés en particular. Este enfoque señala, a grandes rasgos, que la gente no utiliza "rutinas" de acción colectiva que sean desconocidas, sino que aprovecha el aprendizaje de experiencias anteriores. Por ello, cada país, época y situación específica desarrolla sus propias acciones particulares (Tarrow, 1997).

En particular, las acciones llevadas a cabo por los grupos de interés se caracterizan por el hecho de que se expresan de manera diferenciada, es decir, no todos los grupos de interés se constituyen ni expresan de la misma manera. Por ejemplo, muchos de ellos desean interferir en las políticas gubernamentales (como el caso de los sindicatos o movimientos gremiales) mientras que otros grupos simplemente pretenden que el gobierno (o el poder legislativo) favorezca o simpatice con una causa en particular. No obstante, es posible enfatizar que un aspecto distintivo de este tipo de asociación es que siempre se relaciona con el Estado y con el sistema político (Medina, 2009; Kitschelt, 1999).

Dos aspectos finales a considerar respecto de la importancia que puede llegar a tener (o no) un grupo de interés es el tema que este adopte y los recursos que posea. Si el tema es de interés general, lo más probable es que existirán varios grupos de interés o actores sociales e institucionales que querrán influir en las decisiones respecto de ese tema particular, lo que puede restar capacidad de influencia a ciertos grupos al diluir el nivel de adherencia y participación que puedan lograr. Finalmente, los aspectos internos del grupo como los recursos con que cuenta (no solo monetarios), la capacidad de relacionarse con otros actores y su tamaño, son decisivos en la capacidad de influencia que logren. En este sentido, una variable relevante es conocer si el grupo se desenvuelve en el ámbito nacional o a nivel local. Si lo hace de la primera manera será más influyente.

En resumen, lo más relevante de los grupos de interés es que utilizan su capacidad organizativa para influir en la toma de decisiones políticas. Esta influencia, generalmente, es llevada a cabo utilizando los canales institucionales existentes al interior de la sociedad y, por lo mismo, la capacidad de influir por parte de los grupos de interés se ve limitada por los 
aspectos institucionales que rigen a la sociedad, es decir, las características específicas del sistema político y de los mecanismos existentes para la toma de decisiones. En este sentido es importante enfatizar el hecho de que los grupos de interés utilizan principalmente los canales institucionales existentes para establecer sus demandas, a diferencia de los movimientos sociales que funcionan explícitamente fuera de ellos y utilizan estrategias y prácticas que no se insertan en la institucionalidad, como son las marchas y manifestaciones callejeras, llegando incluso al uso de la violencia.

Sin duda, los grupos de interés aportan en términos positivos a la sociedad, pero también presentan aspectos negativos. Entre los aspectos positivos de la existencia de los grupos de interés se encuentra que logran organizar la voluntad de las personas y obtener información acerca de los objetivos de los ciudadanos, a la vez que entregan una oportunidad eficiente de exponer las necesidades de los ciudadanos a los representantes políticos. Sin embargo, su existencia conlleva algunos aspectos negativos, ya que al concitar solo a algunos sectores de la sociedad pueden provocar que ciertas minorías (e incluso algunos grupos mayoritarios no organizados) se vean postergados en sus intereses cuando estos últimos no han logrado la fuerza electoral necesaria para ser representados en el proceso político. Esto es así porque los grupos de interés, al estar organizados, cuentan con otros espacios de influencia que van más allá de lo puramente electoral.

En la actualidad, uno de los grupos de interés más relevante en las economías de mercado son los sectores empresariales y aquellos grupos relacionados a la industria y al comercio (Medina, 2009: 18-20). Si bien estos grupos actúan con la misma lógica que cualquier otro grupo de interés, tienen ciertas particularidades que pueden ser fácilmente identificadas. Olson, en sus estudios acerca de acción colectiva, identifica que: i) el éxito de estos grupos se mide según la utilidad marginal; ii) el sector específico de la asociación económica que participa de las acciones de acción colectiva es crucial para asegurar el éxito en sus gestiones: si los líderes no son respaldados por un número suficiente de miembros, es difícil que sus gestiones lleguen a buen término; y iii) estas asociaciones están organizadas de manera transparente y cuentan con líderes fácilmente identificables, en este sentido existen líderes y seguidores al interior de ellos (Tarrow, 1997: 44).

El poder que tienen los grupos empresariales puede ejemplificarse claramente al observar cifras de Estados Unidos, país que cuenta con estadísticas oficiales asociadas a las acciones de lobby. Durante 1996, las asociaciones de empresarios en conjunto con las asociaciones de profesionales constituyeron más de la mitad de la comunidad de lobistas de Washington y el gasto realizado por ellas representó el $85 \%$ del total del gasto reportado en lobby, superando ampliamente al realizado por otras asociaciones (Baumgartner y Leech, 2001). Si bien en Chile no existen cifras oficiales, las organizaciones empresariales son reconocidas por su nivel de organización y presencia mediática. Entre otras, se puede nombrar a la Cámara de Comercio de Santiago (CCS) y la Cámara Nacional de Comercio, Servicios y Turismo (CNC), la SOFOFA, la CPC y la Asociación de Emprendedores de Chile. Esta última, por medio de su representante Juan Pablo Swett, tuvo un rol relevante durante la discusión de la reforma tributaria. 


\section{IV. ¿POR QUÉ UNA REFORMA TRIBUTARIA CONCITA LA ATENCIÓN DE LOS GRUPOS DE INTERÉS, EN ESPECIAL LA DE LOS GRUPOS EMPRESARIALES?}

Antes de analizar cuál fue el rol de los grupos de interés empresariales en la discusión relativa a la reforma tributaria, es importante reconocer las características que tiene una reforma de este tipo, principalmente porque llama la atención la activa participación de los empresarios en estos temas y no en otro tipo de materias. La razón es que las reformas tributarias, cualquiera sea su extensión y profundidad, concitan gran interés por parte de los diversos actores económicos y sociales, ya que una reforma tributaria provoca grandes efectos sobre la eficiencia y equidad de una sociedad. En este sentido, una reforma tributaria es el campo de la política pública donde hay más intereses en juego y donde más actores participan de manera activa en su diseño, discusión e implementación; por otra parte, una reforma tributaria puede implicar altos costos políticos que pueden recaer sobre el gobierno de turno (o los partidos y coaliciones que lo integran), mientras que los beneficios, en general, se perciben en gobiernos futuros y viceversa; finalmente, de no concitar acuerdos generales, las reformas tributarias pueden constituirse en reformas de corto plazo, dependientes de quien gobierne, con los costos que esta situación conlleva (Stein et al., 2006: cap. 8).

En este sentido, diversos autores estipulan que lo recomendable en el caso de este tipo de políticas en particular es contar con un consenso de todos los actores involucrados incorporando a todos los grupos y agentes, directamente o mediante sus representantes, en las discusiones, los procesos de negociación, el cumplimiento de ciertos acuerdos sobre la forma cómo se realizará la reforma y en el uso que se le dará a los recursos públicos generados (Stein et al., 2006: cap. 8; Lora, 2008). Por lo anterior, la comunicación tanto del contenido de las políticas diseñadas como de la manera en que serán implementadas es crucial. Sánchez (2011) destaca la idea de que la comunicación es uno de los principales ingredientes de la acción política. "Las políticas de comunicación forman parte creciente de la agenda pública de los gobiernos y también de las entidades no estatales y a su vez condicionan la elaboración misma de la agenda" (Sánchez, 2011: 3). Consecuentemente, el análisis técnico puede proporcionar algunas recomendaciones respecto de las opciones óptimas, pero es el análisis político-económico el que aportará la información necesaria acerca del subconjunto de opciones realmente factibles y contribuirá a que se evalúe el costo político de las diversas opciones de reforma. En este sentido, las reformas tributarias hay que pensarlas y entenderlas en su dimensión de contrato social y examinarlas como un elemento constituyente de la relación Estado-sociedad. En el caso de la reforma tributaria recién promulgada, los aspectos relacionados al FUT concitaron la atención de diversos grupos empresariales por las implicancias económicas que su eliminación tendría sobre sus actividades productivas y las utilidades generadas por ellos y por la importancia de esta herramienta como instrumento para financiar futuras inversiones.

\section{V. ¿QUÉ ES EL FUT?}

El mecanismo del FUT nace en los años 80 a raíz de la situación de insolvencia del país, posterior a la crisis económica vivida en esa década, y por tanto obedece a las condiciones 
económicas específicas de ese momento, en especial debido a las restricciones financieras que enfrentaron las empresas en Chile en ese periodo. De esta manera, el FUT fue ideado como una solución para incentivar el ahorro nacional y así permitir un aumento de la inversión y el crecimiento económico al ampliar la capacidad de crédito interno de la economía. Pero a partir de los 90 el país se vuelve más solvente y las grandes empresas acceden al crédito internacional, con lo que la herramienta perdió parte de su sentido original.

En términos prácticos, la Ley de la Renta estableció que los contribuyentes de la primera categoría, que estén obligados o que opten por declarar en dicha categoría sus rentas efectivas, deben Ilevar un libro especial denominado Registro de la Renta Líquida Imponible de Primera Categoría y Fondo de Utilidades Tributables (FUT). En este libro debían consignarse, principalmente, las utilidades no retiradas que genera una empresa, las que al no quedar registradas en la base imponible tiene como resultado que los empresarios no paguen impuestos sobre ellas. Con ello, el sistema tenía como finalidad fomentar el ahorro de las empresas en la forma de utilidades retenidas, fondos que permitirían financiar la inversión futura (Calderón, 2014: 72). El Servicio de Impuestos Internos (SII) estima que, al 2013, habían 247 mil millones de dólares acumulados por concepto del FUT, lo que significa que existen, aproximadamente, US\$50 mil millones de impuestos postergados (Economía y Negocios On-line, 09/06/2014).

Si el FUT fomenta el ahorro y la inversión, ¿cuál es el real problema con este mecanismo que lo hace susceptible de ser eliminado? El problema es que abre la puerta a la elusión tributaria, ya que las utilidades retenidas (FUT), si no se reinvierten, en algún momento son retiradas y pasan a sus respectivos dueños. En ese instante, en que como persona natural deberían pagar $30 \%$ de impuesto, finalmente se paga solo el $10 \%$ porque la empresa "ya pagó" el 20\% restante. Es decir, el FUT funciona como crédito descontando el impuesto ya pagado por la empresa (20\%) en el periodo en que se generaron las utilidades. Por ello el planteamiento original de la reforma tributaria fue que los fundamentos con que se concibió la "estructura de incentivos" del FUT se "han desviado sustancialmente de sus objetivos iniciales y se han convertido en fuentes de elusión e incluso de evasión de impuestos" (Programa de Gobierno de Michelle Bachelet 2014-2018: 25). De este modo, se propone que los dueños de las empresas deberían tributar por la totalidad de las utilidades de sus empresas y no solo sobre las utilidades que retiran (el sistema propuesto operaría sobre la base devengada). En consecuencia, la eliminación del FUT se consideraba una herramienta efectiva para aumentar la recaudación fiscal, ya que la posible elusión tributaria generada por este concepto desaparecería con su fin.

\section{CAMPAÑA DE LOS EMPRESARIOS}

Desde el comienzo de la discusión de la reforma tributaria en el Parlamento, los empresarios se mostraron contrarios a la idea de eliminar el FUT. De hecho, en abril, la CPC encargó dos estudios que analizaron los aspectos económicos y legales de la reforma. En particular, un informe se refirió, exclusivamente, a la eliminación del FUT, proponiendo un mecanismo alternativo para incentivar $y$ facilitar el ahorro por parte de las empresas con el fin de financiar 
sus proyectos de inversión. El argumento central de dicho documento señalaba que con el término del FUT se produciría un perjuicio en la caja de las compañías, limitando el financiamiento para las futuras inversiones (La Tercera Negocios, 27/04/2014). A su vez, se realizó un fuerte lobby en diferentes frentes. En el Congreso, el presidente de la Comisión de Hacienda, Pablo Lorenzini, señaló que durante la discusión de este proyecto no habían estado presentes en los pasillos del Parlamento "los profesionales de siempre", sino una nueva casta. Empresarios de todos los sectores acudieron a la Comisión para expresar sus puntos de vista, desde la CPC hasta Vinos Chile y la Cámara Chilena de la Construcción (CCHC), entre otros. "En temas sectoriales, uno ha visto en el pasado a los lobistas en el Congreso y se ha tomado algún café con ellos. Acá no, porque hemos visto un lobby distinto, de alto nivel. Se trata de expertos que trabajan en universidades e institutos y que han hecho un lobby más bien intelectual. Ahí hay exministros, columnistas, académicos. Algunos de ellos, tengo entendido, han asesorado a los empresarios con estudios que están en un lenguaje bastante preciso y solo para entendidos", explicó (www.reformalareforma.cl, 09/07/2014; The Clinic Online, 14/07/2014)

Como una forma de conocer la fuerza del lobby, The Clinic Online solicitó vía transparencia a los integrantes de la Comisión de Hacienda de la Cámara Alta (Juan Antonio Coloma, José García Ruminot, Ricardo Lagos Weber, Carlos Montes Cisternas, Andrés Zaldívar Larraín) que explicitaran quiénes habían solicitado alguna reunión privada con ellos desde marzo hasta el 1 de julio de 2014. El resultado, como se esperaba, fue que gran parte de quienes pidieron una audiencia son las mismas personas que asistieron a exponer a la Comisión: representantes de grupos empresariales como Andrés Santa Cruz de la CPC, Hermann von Mühlenbrock de la SOFOFA o José Ramón Valente de Econsult. Coloma fue el parlamentario que más reuniones sostuvo, con más de 80, en tanto que Ruminot solo especificó 14 encuentros en el Congreso y cerca de 50 a nivel regional (The Clinic Online, 14/07/2014).

Sin embargo, los empresarios también quisieron influir en la opinión pública realizando campañas de comunicación masiva que dieran a conocer su postura frente a la reforma tributaria. De esta forma nuevos actores aparecieron en escena. Por ejemplo, Bernardo Fontaine coordinó la página $w w w$.reformalareforma.cl financiando un inserto en diversos medios escritos en el que se criticaba duramente el proyecto de ley. El inserto consistió en una recopilación de diversas opiniones técnicas comentando diferentes aspectos de la reforma. La idea era aunar en un grupo a los diferentes movimientos sociales y pequeños gremios en torno a los siguientes lemas: "Esta reforma la pagamos todos", "Pequeñas y Medianas Empresas (PYMES) sí se ven dañadas", "Reforma antiahorro" y "Reforma mal hecha técnicamente". "Queremos ser la voz de los sin voz", dijo en el lanzamiento de su página web. De prosperar este movimiento, la reforma tributaria estaría logrando algo que no se había visto desde hace años en Chile: que los distintos gremios se aglutinen en contra de una idea o proyecto, señalaron algunos líderes empresariales (El Mostrador Mercados, 4/6/2014).

Pero quizás la campaña más comentada y con mayor visibilidad para el público general fue la liderada por Juan Pablo Swett, quien realizó unos cuantos videos a nombre de la Asociación de Emprendedores de Chile, los que fueron subidos a la web. En los videos 
Swett explicaba, en pocos minutos, cómo la reforma tributaria y en particular la eliminación del FUT traerían consecuencias negativas para las PYMES al disminuir la capacidad de endeudamiento de las empresas. Tal como se señaló anteriormente, el FUT es una fuente de financiamiento alternativo para este sector empresarial, el que no siempre ha tenido un acceso fácil al sistema financiero y crediticio formal. De eliminarse el FUT, se elimina esta opción de financiamiento para las nuevas inversiones. "Se adhiere a la necesidad de una reforma tributaria, pero estamos en desacuerdo con la forma en que se va a recaudar", señaló Swett en mayo pasado.

La presión realizada por los empresarios tuvo efecto en el gobierno, el que modificó sustancialmente algunos artículos del proyecto, tocando incluso al Ilamado "corazón de la reforma", concepto acuñado por el Ministro de Hacienda para aludir a aquellos temas en que no se estaba dispuesto a transar, entre ellos la eliminación del FUT. Pero también hubo un efecto a nivel de los partidos políticos y en los parlamentarios. De hecho, la Unión Demócrata Independiente (UDI), tras constatar que los gremios empresariales estaban por llegar a un acuerdo con el gobierno, decidió sumarse a él. De esta manera, el 7 de julio de 2014 se dieron a conocer por la prensa los principales acuerdos entre el gobierno y la oposición que luego darían pie al acuerdo alcanzado en la Comisión de Hacienda con el fin de aprobar la reforma y evitar así que fuera a discusión en la Comisión Mixta. Este acuerdo tributario logrado a "puertas cerradas" por el Ministro de Hacienda con la derecha política contradijo los dichos anteriores emitidos por el secretario de Estado en el sentido de que no se tocaría el corazón de la reforma y por tanto no estaba en negociación la eliminación del FUT. CIPER señaló que "el protocolo debilita el objetivo original: que todos paguen de acuerdo a sus ingresos reales, confirmando que entre quienes hicieron lobby figuran grandes empresarios que también se reunieron con autoridades para atenuar el impacto de la reforma. Y lo lograron" (www.ciperchile.cl, 17/07/2014).

¿Cómo quedó el FUT tras la reforma? El FUT se reemplazará a partir del 1 de enero de 2017. Para ello se crea un sistema semiintegrado de tributación, cuyo funcionamiento es paralelo y opcional al del régimen integrado con atribución de renta, que opera por defecto. De esta manera se modifica el antiguo sistema FUT. Este sistema integrado tiene una tasa de impuestos de primera categoría (a las utilidades de las empresas), que sube gradualmente de $20 \%$ a $25 \%$ en cuatro años. El impuesto pagado seguirá operando como un crédito para los dueños de las empresas al momento de pagar su Impuesto Global Complementario o Adicional (impuesto a las rentas personales).

A su vez, el sistema parcialmente integrado establece un sistema de tasas distintas, según se trate de utilidades invertidas, ahorradas o retiradas por parte de las empresas. Se considera un aumento progresivo del Impuesto de Primera Categoría para las empresas desde $20 \%$ a $25,5 \%$ el primer año de vigencia de la reforma hasta llegar a $27 \%$ el cuarto año. Los empresarios podrán aplicar ese $27 \%$ pagado, como crédito en contra de los impuestos finales, pero solo hasta en $65 \%$ de lo pagado. En términos generales, cuando se retiren utilidades bajo esta modalidad se gravan con Impuesto Adicional del 35\% sobre base retirada, debiendo incluirse en la base la totalidad del Impuesto de Primera Categoría, pero solo el 65\% del 
Impuesto de Primera Categoría pagado constituirá crédito. Esta forma de cálculo determina una tasa de impuesto efectiva de 44,45\%. Los contribuyentes podrán elegir entre estas dos modalidades debiendo permanecer en el sistema elegido al menos cinco años (www.bcn.cl) leyfacil/recurso/reforma-tributaria-2014). En consecuencia, el FUT no se eliminó totalmente y parte del mecanismo existente se mantiene pero con tasas diferentes ${ }^{3}$.

\section{CONCLUSIONES}

Los grupos de interés muchas veces han sido denominados grupos de presión, debido a que su objetivo es influir en el sistema de toma de decisiones de manera de orientar las políticas públicas según sus fines y objetivos. Entre las acciones de influencia que pueden Ilevar a cabo los grupos de interés está el lobby. Por ello su estudio y reglamentación es esencial, debido a las consecuencias negativas en términos de transparencia que esta práctica pueda tener. En Chile, la Ley № 20.730 regula la actividad, si bien aún no entra en vigencia debido a que el reglamento que regula el actuar del gobierno y del Parlamento en este tema se encuentra en etapa de toma de razón en la Contraloría. Una vez finalizado este trámite, la ley y sus reglamentos deberán entrar en vigencia tres meses después de publicados en el Diario Oficial.

No obstante el punto anterior, es posible analizar a la luz de la ley el lobby realizado en el caso de la reforma tributaria, tanto por parte de personeros del gobierno y del Parlamento como de los sectores empresariales. La directora de Incidencia e Investigación de la Fundación Ciudadano Inteligente, María Jaraquemada, señaló que "tanto las acciones realizadas por el gobierno, Renovación Nacional (RN) y la UDI junto a sus equipos técnicos para lograr el protocolo de acuerdo de la reforma tributaria, resultaron plenamente ajustadas con lo que define la ley que regirá la actividad del lobby y de quienes realizan esa función" (www. lanacion.cl, 18/07/2014). En el caso de las tratativas que han hecho los particulares para tratar de influir o para informar de su punto de vista a las autoridades, también caben dentro de la reglamentación del lobby, y en caso que hoy estuviese vigente el articulado los parlamentarios y el gobierno "tendrían que publicar en sus páginas web esas reuniones" (www. nacion.cl, 18/07/2014). En este sentido, el rol del empresario Juan Pablo Swett, presidente de la Asociación de Emprendedores de Chile, quien sostuvo reuniones con senadores de la Comisión de Hacienda, el Subsecretario de Hacienda, Alejandro Micco, y con otros representantes de asociaciones gremiales, fue el de un gestor de intereses particulares al canalizar las inquietudes de las PYMES que lidera sobre los efectos de los cambios tributarios en este sector. Se denomina gestor de intereses particulares al lobista que no recibe un pago por sus gestiones.

Resumiendo, si la normativa hubiese estado vigente, no habrían habido vicios en los encuentros que llevaron a cabo las partes para modificar varios aspectos del texto original y así asegurar los votos de la oposición para el proyecto de ley, de modo que los grupos

3 Diversos medios y actores políticos nombraron "FUTITO" a la manera en que quedó la modificación al FUT, como una forma de ironizar respecto de que el sistema no se alteró radicalmente. 
de interés cumplieron con los objetivos que se habían propuesto de manera adecuada, es decir, sin interferir en la transparencia del proceso (www.lanación.cl, 18/07/2014). No obstante el reconocimiento de la existencia de lobby por parte de los diferentes grupos de interés, uno de los aspectos más difíciles es medir el grado de influencia real de los lobistas. En la actualidad es aceptado que el poder se expresa en múltiples formas, muchas de ellas no son "observables", es decir, el ejercicio del poder no puede cuantificarse únicamente observando las presiones que un legislador recibe por parte de un grupo, o la contribución financiera que realiza un holding empresarial a las arcas de un partido político (Medina, 1999: 6).

A futuro es relevante mejorar en la capacidad para medir el grado de influencia efectiva de los distintos grupos de poder, ya que con este conocimiento será posible establecer acciones tendientes a incrementar los niveles de transparencia, de modo que el lobby tenga una connotación positiva como una forma de participación ciudadana válida y deseable y no la connotación negativa con que cuenta en la actualidad.

\section{BIBLIOGRAFÍA}

Baumgartner, F., J. Berry, M. Hojnacki, C. Kimball y B. Leech (2009): Lobbying and policy change. Who wins, who loses, and why, The University of Chicago Press, Chicago and London.

Baumgartner, F. y B. Leech (2001): "Interest niches and policy bandwagons: patterns of interest group involvement in national politics", The Journal of Politics, 63 (4), pp.1191-1213.

Benítez, S. (2011): “La comunicación estratégica como política pública”, Documento de Opinión, 21, Instituto Español de Estudios Estratégicos.

Bringel, B. (2009): Acción colectiva y democracia: la contribución de Charles Tilly para el debate sobre el ensanchamiento del campo democrático, ponencia presentada en el Eje 1 ("El Estado: agente de gobierno") de las Jornadas Internacionales "Homenaje a Charles Tilly. Conflicto, poder y acción colectiva: contribuciones al análisis sociopolítico de las sociedades contemporáneas", 7-8 de mayo, Madrid.

Calderón, P. (2014): “Fondo de Utilidades Tributables", Revista Tributaria, 10, pp. 69-97.

Cerda, R., J.L Correa, J. Parro y D. Peñafiel (2014): “El Fondo de Utilidades Tributables (FUT): elementos para la discusión", Estudios Públicos, 135, pp. 39-87.

Dalton, R. y M. Wattenberg (eds.) (2000): Parties without partisians. Political change in advanced industrial democracies, Oxford University Press, Oxford.

Ferrando, J. (1977): “Grupos de interés, de promoción y de presión. Institucionalización de los grupos de presión", Revista de Estudios Políticos, 213-214, pp. 9-44.

Gable, R. (1958): "Interest groups as policy shapers", Annals of the American Academy of Political and Social Science, 319, pp. 84-93.

García, J.F. (2008): "Modelos de regulación del lobby en el derecho comparado", Revista Chilena de Derecho, 35 (1), pp. 107-134.

Gómez J.C. y J. O'Farrell (2009): La Economía Política de la política tributaria en América Latina, ponencia presentada en el vigesimoprimer Seminario Regional de Política Fiscal ILPES/CEPAL, 26-29 de enero, Santiago de Chile. 
González, E. (2012): "El proceso de la acción colectiva según Charles Tilly", Ecuador Debate, 87, pp. $51-72$.

Guédez, V. (2009): "Los grupos de interés: de lo transaccional, a lo relacional y a lo consustancial", Boletín CIES, Universidad de Barcelona.

Kitschelt, H. (1999): "Panoramas de intermediación de intereses políticos: movimientos sociales, grupos de interés y partidos a comienzos del siglo XXI", Revista de Investigaciones Políticas y Sociológicas (RIPS), 1 (2), pp. 7-25.

Lachmann, W. (2012): "Grupos de interés, lobbies", Konrad-Adenauer-Stiftung e. V., 9, pp. 5-7.

Levitsky, S. y M. Murillo (2010): "Variación en la fortaleza institucional”, Revista de Sociología, 24, pp. 31-56.

Lijphart, A. (1997): "Unequal participation: democracy's unresolved dilemma", American Political Science Review, 91 (1), pp.1-14.

Lora, E. (2008): "El futuro de los pactos fiscales en América Latina", Documento de Trabajo, 650, Banco Interamericano de Desarrollo (BID), Departamento de Investigación.

Martínez, G. y J. Iranzo (2010): “Charles Tilly: legado y estela. De The Vendée a Contentious Performances, para comprender el conflicto político del s. XIX español", Política y Sociedad, 2010, 47 (2), pp. 195-217.

Medina, I. (2009): “¿Cómo medir la influencia de los grupos de interés? (Propuestas desde el pluralismo, el elitismo y el nuevo institucionalismo)", Working Paper, 279, Institut de Ciències Polítiques i Socials, Universitat Autònoma de Barcelona.

Merino, M. (1995): "La participación ciudadana en la democracia", Cuadernos de Divulgación de la Cultura Democrática, 4, Instituto Federal Electoral, México D.F.

Olson, M. (1992): La lógica de la acción colectiva: bienes públicos y la teoría de grupos, Editorial Limusa-Noriega, México D.F.

Ortiz, L. (2014): "Bondades que presenta el registro FUT para los dueños de empresas chilenas", Revista de Estudios Tributarios, 9, pp. 49-100.

Pérez, G. y A. Natalucci (2008): “Estudios sobre movilización y acción colectiva: interés, identidad y sujetos políticos en las nuevas formas de conflictividad social", en A. Natalucci (ed.): Sujetos, movimientos y memorias. Sobre los relatos del pasado y los modos de confrontación contemporáneos, Al Margen, La Plata.

"Programa de Gobierno de Michelle Bachelet 2014-2018" (2013), disponible en www.michellebachelet. cl/programa/ (recuperado el 05/11/2014).

Ramírez, J. y N. Bravo (2014): “Movimientos sociales en Chile: una radiografía al proceso de movilización 2009-2014", Serie Informe Sociedad y Política, 144, Instituto Libertad y Desarrollo.

Sánchez, S. (2011): "La comunicación estratégica como política pública”, Documento de Opinión, 21, Instituto Español de Estudios Estratégicos.

Sartori, G. (2005): “En defensa de la representación democrática”, en M. Carbonell (comp.): Democracia y representación: un debate contemporáneo, Tribunal Electoral del Poder Judicial de la Federación, México D.F., pp. 21-34.

Stein, E., M. Tomassi, K. Echebarría, E. Lora y M. Payne (2006): La política de las políticas públicas, Planeta, México D.F. 
Tarrow, S. (1997): El poder en movimiento. Los movimientos sociales, la acción colectiva y la política, Alianza, Madrid.

Tilly, C. (1995): “Los movimientos sociales como agrupaciones históricamente específicas de actuaciones políticas", Sociológica, 10 (28), pp. 13-36.

Vargas, S. (2011): "Evaluando la participación social: democracia y políticas públicas", Revista Mexicana de Sociología, 73 (1), pp. 105-137.

\section{Artículos de prensa y páginas web consultadas:}

"Bernardo Fontaine quiere dejar el vuelo en solitario y convertirse en referente" (04/06/2014), www. elmostradormercados.Cl (recuperado el 22/11/2014).

"Empresarios critican en Washington reforma tributaria" (30/06/2014), www.lasegunda.com (recuperado el 01/12/2014).

Ganora, E. y M.E. Álvarez (29/04/2014): “Gobierno lanza campaña para defender reforma tributaria: La Moneda 'viralizó' video que busca contrarrestar críticas", www.latercera.cl (recuperado el $12 / 11 / 2014)$.

"Impuestos que se han postergado con el FUT suman US\$50 mil millones a de junio de 2013" (09/06/2014), www.economiaynegocios.cl (recuperado el 18/11/2014).

Jimeno, P. y M. Sánchez (27/04/2014): “El camino de los empresarios para abordar la reforma tributaria en el Senado", www.latercera.cl.

"Los empresarios no creen que la reforma tributaria introduzca incentivos al ahorro" (04/05/2014), www.economiaynegocios.cl (recuperado el 22/11/2014).

Saure, G. (18/07/2014): "Ciudadano Inteligente: lobby por reforma tributaria se ajustó a la ley", www. lanacion.cl.

Skoknic, F. (17/07/2014): "Las huellas del lobby en la reforma tributaria: los forados a favor de la elusión que dejó el acuerdo tributario de Arenas con la derecha", www.ciperchile.cl (Actualidad y Entrevistas) (recuperado el 18/11/2014).

www.bcn.cl/leyfacil/recurso/ley-del-lobby (recuperado el 01/12/2014).

www.bcn.cl/leyfacil/recurso/reforma-tributaria-2014 (Guía legal sobre la reforma Tributaria 2014) (recuperado el 09/11/2014).

www.cpc.cl (recuperado el 02/12/2014 y el 15/03/2015).

www.michellebachelet.cl/programa/ (Programa de Gobierno de Michelle Bachelet 2014-2018)

www.sii.cl (recuperado el 15/11/2014).

Recibido: 23-03-2015

Aceptado: 11-05-2015 